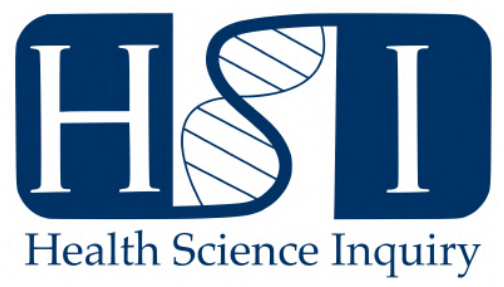

\title{
Present State of Brain Machine Interfaces
}

\author{
Kartik Pradeepan ${ }^{1}$ \\ ${ }^{1}$ Department of Neuroscience, University of Western Ontario
}

As early as the 1700s, scientists have had an idea that the nervous system uses electrical activity. In the time since this idea sparked, the fantasy of manipulating this electrical force has been a major plot point of several famous novels, and the goal of many therapies and medical treatments. Additionally, in more recent years, our world has become awash with electrical devices and technology so much so that our actions and thoughts "are increasingly becoming shaped and substantiated by machines" [1]. Through the blurring of this line between humans and machines, Marshall McLuhan, a philosopher, describes "technology and media as extensions of our central nervous system" [2]. Nowhere is this blurring more explicitly evident than in the technological interfacing of the brain and machine, known as brain-machine or brain-computer interfaces (BMI or BCI, respectively).

The simplest definition of a brain-machine interface (BMI) is the functional linking of neural ensembles directly with a man-made machine. BMIs do this through sensing/acquiring brain signals, analyzing them, and then translating the signals into commands that are relayed to an output machine to carry out a specific intended action [3]. BMIs are commonly divided into three types: sensory (e.g. cochlear implants), motor (e.g. neural-limb prosthetic) or cognitive (i.e. re-establishing proper neural interactions within the brain) [4]. It is important to note that while BMIs are recording information from the brain, they are not mind-reading devices. Rather, the user and the BMI work together, first by training to use the BMI so that the brain is able to generate signals of intention, and then by translating the commands to an output machine to carry out that user's intention [3].

BMI systems typically consist of "four sequential components: 1) signal acquisition, 2) feature extraction, 3) feature translation, and 4) device output" [3]. Signal acquisition is recording of brain signals through various approaches such as electroencephalography (EEG) and electrocorticography (ECoG) [3]. To acquire brain signals, two types of cortical recordings are typically considered. Action potentials are the functional units of the nervous system and these recordings are crucial for the precision of certain neuroprosthetics. Local field potentials, which are more commonly used, are the result of coordinated activity of many neurons and these recordings are the driving forces behind the neuroprosthetics [5]. The signal, once acquired, is amplified and filtered to improve the signal-to-background noise ratio so it can be electronically processed. Feature extraction involves analyzing the digital signal and identifying important signal features, like those related to a person's intent. Feature translation then takes the identified signal features and runs it through a translation algorithm to convert it to specific commands for the output device. Finally, the commands generated operate the device, allowing the user to intentionally control the external device using their brain. While the precise signal may vary between motor, sensory and cognitive BMIs, the four components are typically conserved one way or another [3].

Although considerable effort has been made in motor BMIs, advancements that aim to fix more complex sensory modalities, like restoring basic elements of visual perception, are not yet considered useful enough to justify the costs or risks to the patient [5]. A reason for the slower progress of sensory BMIs is because sensory systems are composed of hierarchical processing areas that flow from lower-order systems to higher-order areas, but also feeds back to the lower-order systems. Additionally, sensory impairments can occur anywhere along this chain of processing areas, to give rise to a complete loss of sensation to deficits in components of higher-level sensory processing. For example, patients with lesions to the primary visual cortex may have cortical blindness where they're unable to consciously perceive visual stimuli but can still subconsciously utilize visual information [6]. It is because of the complexity in sensory processing and circuitry between neural ensembles that advancements in sensory and cognitive BMIs have not been as significant as seen in motor BMIs.

Evidently, there are certainly a few challenges to current BMI technology, with some being critical bottlenecks that need to be overcome in order to seamlessly integrate any output device with the user. Despite this, the increasing numbers of researcher groups and companies investing into the area as well as improvements in other related disciplines suggest positive growth and further advancements [7]. Beyond perfecting neuroprosthetics, BMIs offer the potential to revolutionize the human experience through human enhancement but may also offer a novel understanding on consciousness and philosophy of the mind. 


\section{REFERENCES}

[1] Nair P. Brain-machine interface. Proceedings of the National Academy of Sciences. 2013;110(46):18343-18343.

[2] McLuhan M. Understanding Media: The Extensions of Man. New York: The New American Library. Inc; 1964.

[3] Shih JJ, Krusienski DJ, Wolpaw JR. Brain-computer interfaces in medicine. In: Mayo Clinic Proceedings. vol. 87. Elsevier; 2012. p. 268-279.

[4] Sanchez JC, Principe JC. Brain-machine interface engineering. Synthesis Lectures on Biomedical Engineering. $2007 ; 2(1): 1-234$.
[5] Langmoen IA, Berg-Johnsen J. The brain-computer interface. World neurosurgery. 2012;78(6):573.

[6] Lebedev M. Brain-machine interfaces: an overview. Translational Neuroscience. 2014;5(1):99-110.

[7] Ramadan RA, Vasilakos AV. Brain computer interface: control signals review. Neurocomputing. 2017;223:2644 .

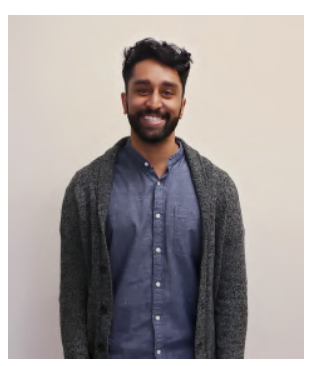

Kartik completed his undergraduate degree at University of Western Ontario with a Double Major in Genetics and Physiology. He is currently pursuing a Master's in Neuroscience at the university in Dr. Julio Martinez-Trujillo's cognitive neurophysiology lab. His project focuses on a computational approach to understanding how iPSC-derived neuronal networks differentially develop as a result of various mutations that are known and believed to contribute to Autism Spectrum Disorder. 